Check for updates

Cite this: RSC Adv., 2017, 7, 45101

Received 28th July 2017

Accepted 15th September 2017

DOI: $10.1039 / c 7 r a 08343 b$

rsc.li/rsc-advances

\section{Passivation of high aspect ratio silicon nanowires by using catalytic chemical vapor deposition for radial heterojunction solar cell application}

\author{
Gangqiang Dong, Yurong Zhou, (D) * Hailong Zhang, Fengzhen Liu, (D) * Guangyi Li \\ and Meifang Zhu
}

\begin{abstract}
High aspect ratio silicon nanowires (SiNWs) prepared by metal-assisted chemical etching were passivated by using catalytic chemical vapor deposition (Cat-CVD). Film conformality of hydrogen amorphous silicon (a-Si : H) deposited by Cat-CVD on SiNWs was investigated and a relationship between the average length of SiNWs and the deposition time of a-Si : $\mathrm{H}$ was determined. By optimizing atomic $\mathrm{H}$ treatment and a- $\mathrm{Si}: \mathrm{H}$ deposition on $1 \mu \mathrm{m}$ length silicon nanowires, a-Si : H/SiNWs radial hetero-junction (RJ-HET) solar cells were fabricated and an efficiency of $16.02 \%$ was achieved. Compared with radial homo-junction (RJ-HOMO) solar cells and planar hetero-junction (P-HET) solar cells, the improvements of open circuit voltage and short circuit current of RJ-HET solar cells are discussed based on measurements of capacitance-voltage, dark current-voltage and quantum efficiency. The RJ-HET solar cell based on the SiNW array also shows better omni-directional antireflection properties than the P-HET solar cell.
\end{abstract}

\section{Introduction}

Radial $\mathrm{p}-\mathrm{n}$ junction solar cells $\mathbf{s}^{1,2}$ based on vertically aligned silicon nanowires (SiNWs) set carrier collection in a direction perpendicular to light absorption. Besides the natural and $j$ excellent light-trapping ability of the nanowire structure, radial $\mathrm{p}-\mathrm{n}$ junctions show an advanced electric collection mode due to a short carrier collection distance, which is beneficial to solar cell performance. Meanwhile, by using the radial $\mathrm{p}-\mathrm{n}$ junctions, competitive efficiencies can likely be maintained for ultrathin crystalline silicon solar cells when shrinking the volume of silicon and using poor quality silicon absorber. Therefore, more and more studies have been conducted on SiNWs based radial $\mathrm{p}-\mathrm{n}$ junction solar cells.

In 2007, Tian et al. ${ }^{3}$ first reported a coaxial single SiNW based radial $\mathrm{p}-\mathrm{i}-\mathrm{n}$ solar cell prepared by vapor-liquid-solid technique which obtained a conversion efficiency of $3.4 \%$. After that, with application of lithography and high temperature diffusion ${ }^{4,5}$ or combination of metal-assisted chemical etching (MACE) and low temperature catalytic shallow doping, ${ }^{6}$ radial $\mathrm{p}-\mathrm{n}$ junction concept has been implemented on SiNWs arrays and reported efficiency increased up to $>13 \%$. However, the reported performances can still hardly meet people's expectation. On the other hand, the radial junctions are usually in a radial homojunction (RJ-HOMO) status. ${ }^{7,8}$ The intensified interfacial recombination brought by increased surface area of SiNWs is not well handled.

University of Chinese Academy of Sciences, Beijing 100049, China. E-mail: zhouyurong@ucas.ac.cn; liufz@ucas.ac.cn
As a result, a common feature of these RJ-HOMO solar cells is the relatively low open circuit voltage $\left(V_{\mathrm{oc}}\right)$.

Optimized surface passivation is critical to obtain higher efficiency of SiNWs solar cells. In the case of silicon heterojunction solar cells ${ }^{9,10}$ (abbreviated to HIT, SHJ or HET solar cells), it's well known that c-Si surface is well passivated by hydrogenated amorphous silicon (a-Si : H) thin film and $V_{\text {oc }}$ larger than $740 \mathrm{mV}$ can be achieved. Recently, radial heterojunction (RJ-HET) solar cells ${ }^{11-13}$ with a-Si : $\mathrm{H}$ thin films functioned as passivation layer and emitter were successfully fabricated on MACE SiNWs. Plasma enhanced chemical vapor deposition (PECVD), a mature thin film deposition technology widely used in commercial industry, is commonly adopted to deposit silicon thin films. Reported $V_{\mathrm{oc}}$ and conversion efficiency of RJ-HET solar cells continuously improved and exceeded RJ-HOMO solar cells ${ }^{14,15}$ in a short time. However, interface and growth layers have to experience ion bombardment during PECVD process, thus surface damage is unavoidable. And, conformal coverage of small surface features with high aspect ratio is hard to accomplish due to the correlation between surface morphology and local potential field. ${ }^{16}$ For this reason, PECVD cannot provide good interface passivation for MACE SiNWs with high aspect ratio (usually $>10$ ) and large density.

Catalytic chemical vapor deposition (Cat-CVD), ${ }^{17}$ also called hot wire CVD (HWCVD), is greatly different form PECVD technology. In Cat-CVD, reactive gases (e.g. silane and hydrogen) are catalytically dissociated on surface of heated filament with very high efficiency. The merits of Cat-CVD ${ }^{18-20}$ are conformal coverage of thin film on small objects, out of surface damage caused by ion bombardment and large amount of atomic 
hydrogen. Compared with PECVD, Cat-CVD can provide better surface passivation which leads to less shunts at the a-Si : H/ SiNWs interface. And, film deposition process of Cat-CVD with plasma-free is easier to control than PECVD. Cat-CVD has been used to create many novel thin films and devices (e.g. $\mathrm{SiN}_{x}$ : H passivation layer in multi-crystalline Si solar cells, polymer materials serving as water vapor barrier layers, thin film silicon solar cells, HIT solar cells etc.) and it is gradually developed for wider application for commercial implements. ${ }^{16,17,21}$

In this paper, a-Si : $\mathrm{H}$ shells were deposited by Cat-CVD technology on surface of MACE SiNWs to construct a-Si : H/ SiNWs RJ-HET solar cells. According to the SiNWs with varied lengths, conformality and passivation effect of a-Si : H layers were investigated. Conversion efficiency $>16 \%$ was achieved for a-Si : H/SiNWs RJ-HET solar cells. Performance of a-Si : H/ SiNWs RJ-HET solar cells are presented with discussion of interface passivation quality, possible radial transport of carriers and light trapping in omnidirectional angle.

\section{Experimental details}

Polished (100) oriented silicon wafers (p-type, resistivity 1-3 $\Omega$ $\mathrm{cm}$ ) were used in this work to investigate passivation properties and construct RJ-HET solar cells. The wafers were cleaned in ethanol for 10 minutes and acetone for 5 minutes in an ultrasonic machine at room temperature. Then the wafers were dipped into $2 \%$ HF solution for 1 minute to remove native oxide layer. After this, the cleaned silicon wafers were immersed into an aqueous solution which containing $5 \mathrm{~mol} \mathrm{~L}^{-1} \mathrm{HF}$ and $0.02 \mathrm{~mol} \mathrm{~L}^{-1} \mathrm{AgNO}_{3}$ to prepare SiNWs array. After etching, the as-prepared samples were rinsed copiously in deionized water and then dipped into a $\mathrm{NH}_{3} \cdot \mathrm{H}_{2} \mathrm{O}: \mathrm{H}_{2} \mathrm{O}_{2}(\mathrm{v} / \mathrm{v}=3: 1)$ solution for 5 minutes to remove the $\mathrm{Ag}$ layer which wrapped on SiNWs arrays. In the end, the SiNWs samples went through standard RCA clean procedure.

The schematic diagram of fabricated RJ-HET solar cells is shown in Fig. 1. After thermal evaporation of aluminum on rear surface, thermal oxidation was processed at $930{ }^{\circ} \mathrm{C}$ with mixed

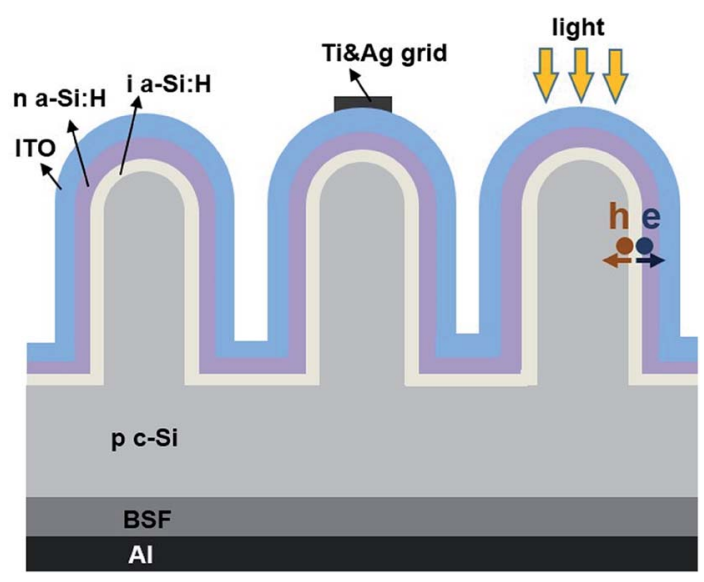

Fig. 1 Cross-section schematic of RJ-HET solar cell. gases of $\mathrm{N}_{2}$ and $\mathrm{O}_{2}$ for 1 hour to form the $\mathrm{Al}$ back surface field (BSF). Then, 10\% HF solution was used to remove oxide layer of front surface. Before depositing intrinsic passivation layer (i a-Si : H) and phosphorus doped emitter (n a-Si : H), atomic hydrogen treatments were proceeded in Cat-CVD chamber for 60 seconds. The temperatures of the hot filaments (Ta wires, $0.5 \mathrm{~mm}$ diameter) and the substrate were kept at $1750{ }^{\circ} \mathrm{C}$ and $150{ }^{\circ} \mathrm{C}$, respectively. Other parameters are listed in Table 1.

After the formation of a-Si:H/SiNWs hetero-junction, a $120 \mathrm{~nm}$ ITO layer (40 $\Omega \square^{-1}$ ) was deposited by reactive evaporation in a deposition rate of $0.5 \mathrm{~nm} \mathrm{~s}^{-1}$ on top of the solar cell to make electrical contacts. The top grid was prepared by $\mathrm{Ti}$ and $\mathrm{Ag}$ evaporation and the back electrode was formed by $\mathrm{Al}$ evaporation.

A conventional planar hetero-junction (P-HET) solar cell was prepared on polished wafer under the same procedure as RJHET solar cell. A RJ-HOMO solar cell was prepared on SiNWs by low temperature catalytic shallow diffusion technology ${ }^{6}$ carried out in the Cat-CVD chamber under a substrate temperature of $250{ }^{\circ} \mathrm{C} . \mathrm{H}_{2}$-diluted $\mathrm{PH}_{3}(0.5 \%)$ was used as the doping gas. After a 25 min doping, a junction depth of about $10 \mathrm{~nm}$ can be formed and a radial-homojunction structure can be obtained on the SiNWs. The P-HET and P-HET solar cells were set as comparison samples.

The surface morphologies of a-Si : H/SiNWs samples were observed by using scanning electron microscope (Hitachi S4800). The minority carrier lifetime was measured by Semilab WT-1200. The measurement of capacitance-voltage $(C-V)$ was carried out on a computer-controlled HP4192A impedance analyzer at $1 \mathrm{MHz}$ fixed frequency with AC voltage of $10 \mathrm{mV}$. The illuminated current density-voltage characterization of the solar cells was measured under AM1.5 $\left(100 \mathrm{~mW} \mathrm{~cm}^{-2}\right.$, at $\left.25^{\circ} \mathrm{C}\right)$ illumination.

\section{Results and discussion}

\subsection{Conformality of a-Si : $\mathrm{H}$ layer deposited onto SiNWs by Cat-CVD}

The conformal coverage of a-Si : $\mathrm{H}$ thin film onto SiNWs array plays an essential role of reducing surface recombination and forming good radial pn junctions with fewer shunts. In Fig. 2, SEM images of SiNWs arrays with varied average length after aSi : $\mathrm{H}$ film deposition with different thickness are shown. Before the a-Si : $\mathrm{H}$ deposition, atomic $\mathrm{H}$ treatments were carried out for about $60 \mathrm{~s}$. Compare Fig. 2(a) and (b), of which share the same $50 \mathrm{~s}$ of a-Si : $\mathrm{H}$ deposition time, the $1 \mu \mathrm{m}$-length SiNWs sample shows a better uniform coating of a-Si : $\mathrm{H}$ along the nanowires than the $500 \mathrm{~nm}$-length nanowires. The $500 \mathrm{~nm}$ length SiNWs sample presents wider diameter on top of nanowires. From these we know that $20 \mathrm{~nm}$ thick (50 s deposition) a$\mathrm{Si}$ : $\mathrm{H}$ is optimal for $1 \mu \mathrm{m}$-length SiNWs sample, meanwhile, the $50 \mathrm{~s}$ of a-Si : $\mathrm{H}$ deposition time is over long for $500 \mathrm{~nm}$-length nanowires. It must be pointed out that the thickness we mentioned here refers to the thickness of a-Si : $\mathrm{H}$ deposited on polished Si wafer at the same condition. Due to much larger surface area of SiNWs, under the same deposition condition, a$\mathrm{Si}: \mathrm{H}$ thin film is much thinner on SiNWs than on flat Si wafer. 
Table 1 Parameters for atomic $\mathrm{H}$ treatment and a-Si : $\mathrm{H}$ thin films deposition by Cat-CVD

\begin{tabular}{lcllr}
\hline Process & $\mathrm{H}_{2}(\mathrm{sccm})$ & $\mathrm{SiH}_{4}(\mathrm{sccm})$ & $\mathrm{PH}_{3}(\mathrm{sccm})$ & \multicolumn{1}{c}{ Pressure $(\mathrm{Pa})$} \\
\hline Atomic H treatment & 20 & - & - & 10 \\
i a-Si:H deposition & 3 & 1 & 0.02 & 2 \\
n a-Si:H deposition & 3 & 1 & 1
\end{tabular}

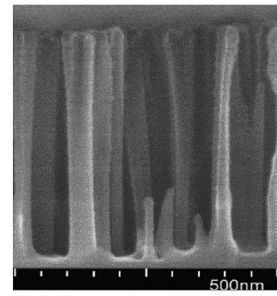

(a)

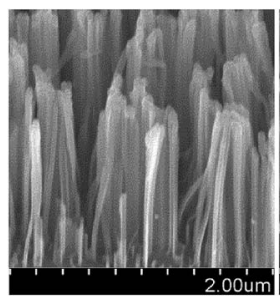

(d)

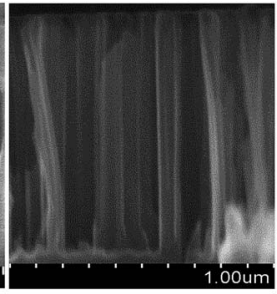

(b)

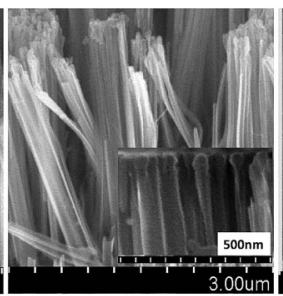

(e)

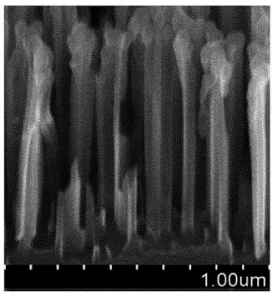

(c)

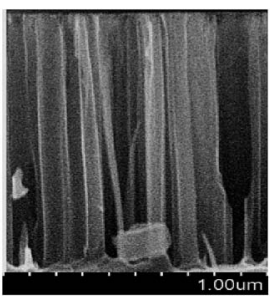

(f)
Fig. 2 Cross-sectional SEM images of a-Si: H/SiNWs with varied average length of SiNWs and different thickness of a-Si : $\mathrm{H}$ thin films. Different thickness was obtained by controlling the deposition time. The combination of average length of SiNWs and thickness (or deposition time) of a-Si : $\mathrm{H}$ is (a) $500 \mathrm{~nm}$ and $20 \mathrm{~nm}$ (50 s), (b) $1 \mu \mathrm{m}$ and $20 \mathrm{~nm}(50 \mathrm{~s}),(\mathrm{c}) 1 \mu \mathrm{m}$ and $60 \mathrm{~nm}(150 \mathrm{~s}),(\mathrm{d}) 2 \mu \mathrm{m}$ and $60 \mathrm{~nm}$ (150 s) and (e) $3 \mu \mathrm{m}$ and $60 \mathrm{~nm}(150 \mathrm{~s})$. A high magnified image of top of nanowires is attached in (e). The deposition pressure of a-Si : $\mathrm{H}$ was fixed at $2 \mathrm{~Pa}$. A cross-sectional SEM image of $1 \mu \mathrm{m}$-length SiNWs without a-Si : $\mathrm{H}$ coating is shown as a reference in (f).

When a-Si : $\mathrm{H}$ deposition time extends up to $150 \mathrm{~s}(60 \mathrm{~nm}$ thickness) for $1 \mu \mathrm{m}$-length SiNWs, thicker layer of a-Si : $\mathrm{H}$ is accumulated on top of nanowires and forms many "fat heads", as shown in Fig. 2(c). If we solely increase the average length of SiNWs to $2 \mu \mathrm{m}$ (Fig. 2(d)) and $3 \mu \mathrm{m}$ (Fig. 2(e)), those "fat heads" gradually shrink.

The above phenomena indicate that, in order to realize conformal coverage of a-Si : $\mathrm{H}$ on nanowires, there is an optimal combination of a-Si : $\mathrm{H}$ thickness and average length of nanowires. Thicker a-Si film (or longer deposition time) is needed for longer nanowires to form uniform coating and vice versa. When deposition time (or thickness) of a-Si : $\mathrm{H}$ and average length of SiNWs match, conformal cover can be realized, as Fig. 2(b) shows. This phenomenon also proves that during Cat-CVD process which has plenty of atomic $\mathrm{H}$ in the gas phase, adsorbed radicals diffuse ${ }^{22}$ along hydride terminated surface of nanowires. In the high magnified image in Fig. 2(e), thin neck just under small "head" of each nanowire can be determined. This is probably due to the radicals depletion caused by long distance diffusion toward the bottom of nanowire. However, once side wall of SiNWs covered by a-Si : $\mathrm{H}$ thin film, long distance diffusion will be disturbed by increased surface roughness, and then "fat heads" appear due to the shadow effect as Fig. 2(c) shows. ${ }^{23}$

It's worth noting that radials may not reach the bottom of long SiNWs at relatively low substrate temperature $\left(150{ }^{\circ} \mathrm{C}\right)$. Furthermore, bending and aggregating appear on nanowires with excess length, which increases difficulties of uniform coating of a-Si : H. Although long nanowires are good for light absorption, with the comprehensive consideration of conformality of a-Si : $\mathrm{H}$ layer, $1 \mu \mathrm{m}$-length SiNWs were finally chosen to prepare solar cells. In this case, when the thickness of a-Si : $\mathrm{H}$ is in range of $20 \mathrm{~nm}$ and $60 \mathrm{~nm}$, fairly conformal coverage can be achieved. Now, a nice passivation characteristic of a-Si : H/SiNWs interface is possible. As we expected, after the a-Si : $\mathrm{H}$ deposition for about $50 \mathrm{~nm}$, effective minority carrier lifetime $\left(\tau_{\text {eff }}\right)$ of the $1 \mu \mathrm{m}$-length SiNWs sample is effectively improved from $80 \mu \mathrm{s}$ (as-cleaned) to $160 \mu \mathrm{s}$. Furthermore, radial transportation and collection of carriers can be realized by depositing an ITO layer on SiNWs.

\subsection{Optimization of the RJ-HET solar cells}

In this work, intrinsic a-Si : $\mathrm{H}$ thin film deposited by Cat-CVD functioned as passivation layer and $\mathrm{n}$ type a-Si : $\mathrm{H}$ thin film deposited by Cat-CVD functioned as emitter layer. Taking into account of the total thickness of a-Si : $\mathrm{H}$ required by conformal cover, two series of RJ-HET solar cells with the structure of grid/ ITO/n a-Si : H/i a-Si : H/p c-Si/Al were fabricated to optimize the thickness of intrinsic and $\mathrm{n}$ type a-Si : $\mathrm{H}$ thin films, respectively. In series 1 , thickness of $\mathrm{n}$ type a-Si : $\mathrm{H}$ was fixed at $20 \mathrm{~nm}$ and thickness of intrinsic a-Si : $\mathrm{H}$ was varied from 0 to $15 \mathrm{~nm}$. The illuminated $I-V$ data including open circuit voltage $\left(V_{\mathrm{oc}}\right)$, short circuit current density $\left(J_{\mathrm{sc}}\right)$, fill factor (FF) and efficiency (EFF) of samples in series 1 are plotted in Fig. 3(a). $V_{\text {oc }}$ rises monotonically with the thickness of intrinsic a-Si : $\mathrm{H}$, which indicates that thicker $\mathrm{i}$ a-Si : $\mathrm{H}$ coverage, less shunts. When $\mathrm{i}$ a-Si : $\mathrm{H}$ is above $10 \mathrm{~nm}, J_{\mathrm{sc}}$ and FF start to decrease due to light absorption and carriers recombination in the intrinsic a-Si : $\mathrm{H}$ layer. In series 2, thickness of intrinsic a-Si : $\mathrm{H}$ was fixed at optimized value of $10 \mathrm{~nm}$ and thickness of $\mathrm{n}$ type a-Si : $\mathrm{H}$ was tuned from $5 \mathrm{~nm}$ to $30 \mathrm{~nm}$. The light $I-V$ data of samples of series 2 are plotted in Fig. 3(b) and the optimized thickness of $\mathrm{n}$ a-Si : $\mathrm{H}$ is $20 \mathrm{~nm}$. The optimal overall thickness of the intrinsic and $\mathrm{n}$ type a-Si : $\mathrm{H}$ is around $30 \mathrm{~nm}$, which is consistent with previous SEM measurements. Usually, the overall thickness of a-Si : $\mathrm{H}$ layers is 10-15 nm on flat substrate. However, thicker a-Si : $\mathrm{H}$ layers (determined from flat surface condition) are needed to realize optimal conformal coverage of SiNWs with enlarged surface 
(a)

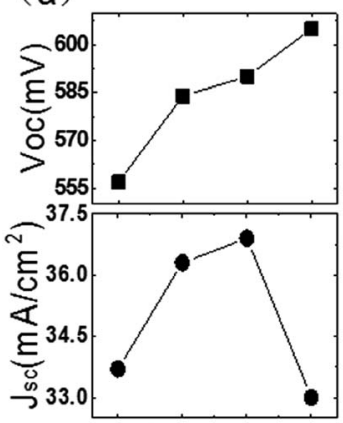

(b)

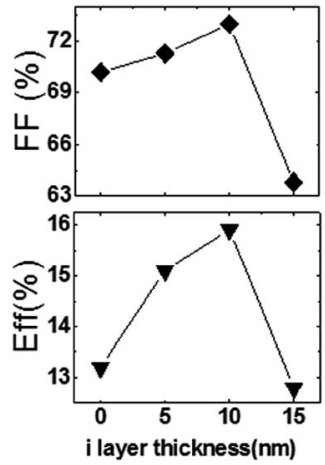

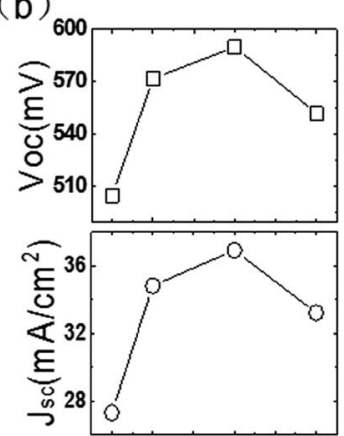
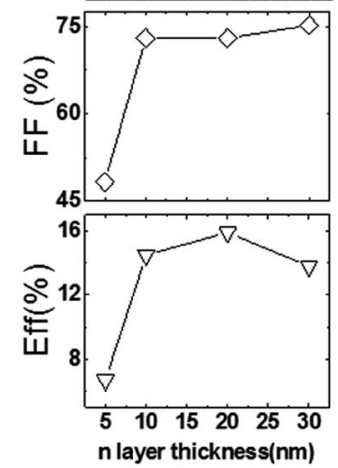

Fig. 3 Light $I-V$ data of RJ-HET solar cells with the structure of grid/ ITO/n a-Si:H/i a-Si:H/p c-Si/Al. (a) Series 1 with tuned thickness of intrinsic a-Si : $\mathrm{H}$ layer. (b) Series 2 with tuned thickness of $\mathrm{n}$ type a$\mathrm{Si}: \mathrm{H}$ layer.

area. Finally, a highest efficiency of $15.9 \%$ was achieved for the two series of RJ-HET solar cells.

\subsection{Comparation of the solar cells with different structures}

To further understand the passivation effect of Cat-CVD on nanostructure, solar cells with different structures, including PHET, RJ-HOMO and RJ-HET, were fabricated and compared. Besides c-Si substrate, back and front contacts went though the same fabrication process and optimized deposition condition was adopted for intrinsic a-Si : $\mathrm{H}$ layer for all solar cells. The highest light $I-V$ data of each solar cell structures are summarized in Table 2. P-HET solar cell has the highest $V_{\mathrm{oc}} \cdot V_{\mathrm{oc}}$ of RJ-HET solar cell is $12 \mathrm{mV}$ lower than P-HET solar cell, but $69 \mathrm{mV}$ higher than RJ-HOMO solar cell. Since RJ-HOMO soalr cell and RJ-HET solar cell are both based on $1 \mu \mathrm{m}$-length SiNWs, they have similar $J_{\mathrm{sc}}$ which is much higher than P-HET solar cell. RJ-HET solar cell has the highest efficiency of $16.02 \%$ of these three structures.

Table 2 Parameters of the optimized P-HET, RJ-HOMO and RJ-HET solar cells: $V_{\mathrm{oc}}, J_{\mathrm{sc}}, \mathrm{FF}$, and EFF. Solar cell with highest efficiency was selected for each structure

\begin{tabular}{lllll}
\hline & $V_{\text {oc }}(\mathrm{mV})$ & $J_{\text {sc }}\left(\mathrm{mA} \mathrm{cm}^{-2}\right)$ & FF $(\%)$ & EFF (\%) \\
\hline P-HET & 606 & 29.42 & 76.75 & 13.68 \\
RJ-HOMO & 525 & 36.61 & 73.13 & 14.05 \\
RJ-HET & 594 & 36.98 & 72.93 & 16.02
\end{tabular}

For better understand of interface properties of these solar cell structures, capacitance vs. voltage $(C-V)$ and dark $J-V$ measurements were carried out for P-HET, RJ-HOMO and RJHET solar cells. Fig. 4(a) shows the relationship of $W^{2}$ ( $W$, depletion region width) and $V$ which were re-plotted from the $C-V$ measurements in reverse bias condition. The magnitude of effective density of localized gap states of the interface layer $\left(N_{\mathrm{I}}\right)$ can be estimated from the slope of $W^{2}-V$ curves. ${ }^{24}$ It can be seen that P-HET solar cell has the smallest $N_{\mathrm{I}}$ due to good passivation of interface. Both RJ-HOMO and RJ-HET solar cells present higher $N_{\mathrm{I}}$ than P-HET, which can be assigned to larger surface area induced by SiNWs. $N_{\mathrm{I}}$ of RJ-HET solar cell is one magnitude lower than RJ-HOMO solar cell, which confirms the benefits of a-Si : H/c-Si heterojunction structure.

Dark $J-V$ characteristics of these three types of solar cells are depicted in Fig. 4(b). The diode quality factor $n_{2}$ and the saturation current density $J_{02}$ were fitted from the dark $J-V$ curves in the forward voltage region of $0.15<V<0.3 \mathrm{~V}$ based on the double diode model. ${ }^{25}$ RJ-HOMO solar cell has the highest $n_{2}$ (3.62) and $J_{02}\left(7.42 \times 10^{-6} \mathrm{~A} \mathrm{~cm}^{-2}\right)$, which is probably due to serious surface recombination. In comparison, $n_{2}$ is reduced to 2.24 and $J_{02}$ is reduced to $1.07 \times 10^{-6} \mathrm{~A} \mathrm{~cm}^{-2}$ for the RJ-HET solar cell also fabricated with SiNWs wafer. Similar phenomenon can be observed in reverse voltage region of dark $J-V$ curves. RJ-HOMO solar cell has the highest leakage current density, while leakage current density of RJ-HET solar cell is much lower.

Both $C-V$ measurements and dark $J-V$ characteristics indicate that interface condition of RJ-HET structure is much better than that of RJ-HOMO structure. In RJ-HET solar cell, lower density of defects states in energy band gap lead to decreased recombination and higher $V_{\mathrm{oc}}$. According to the average data of the light $I-V$ of around 5 parallel samples, $V_{\text {oc }}$ of RJ-HET solar cells $(581 \pm 13 \mathrm{mV}$ ) increased by about $50 \mathrm{mV}$ compared with that of RJ-HOMO solar cells $(530 \pm 15 \mathrm{mV})$. P-HET solar cells have the best interface condition and the highest $V_{\text {oc }}(596 \pm$
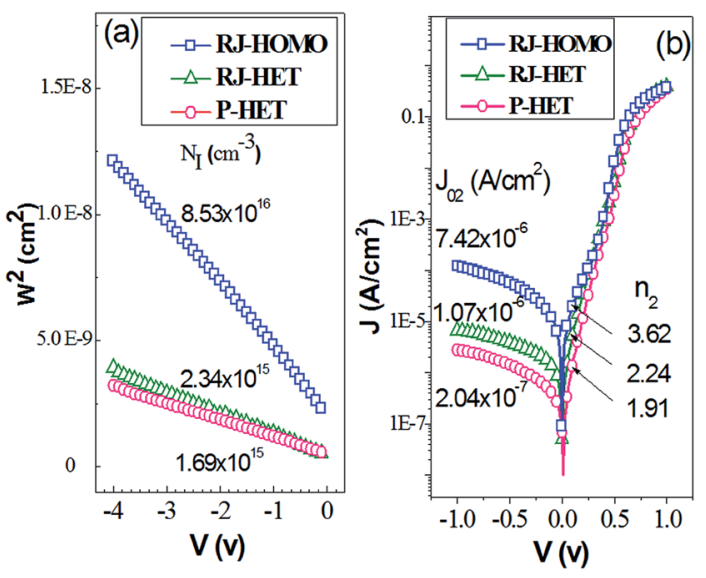

Fig. 4 Square of width of the depletion region as a function of voltage obtained from the $C-V$ measurement (a) and dark $J-V$ characteristics (b) of these three solar cells. Effective density of localized gap states $\left(N_{1}\right)$, diode quality factor $\left(n_{2}\right)$ and saturation current density $\left(J_{02}\right)$ of these three solar cells are given in the figures. 
$10 \mathrm{mV}$ ) as expected, mainly due to the smooth interface and good a-Si : $\mathrm{H}$ passivation by Cat-CVD.

From Table $2, J_{\mathrm{sc}}$ of SiNWs solar cells (RJ-HET and RJ-HOMO) increases 24-25\% compared with that of the P-HET solar cell. Excellent light trapping effect of the SiNWs structure contributes to higher $J_{\mathrm{sc}}$. However, average reflectance $(350-1000 \mathrm{~nm}$ ) of ITO covered SiNWs is $8.1 \%$ and ITO covered polished $\mathrm{c}-\mathrm{Si}$ is $16.6 \%$. So, decrease of average reflectance caused by the SiNWs structure is not the only factor that make $J_{\text {sc }}$ increase of $24-25 \%$.

For radial junction SiNWs solar cells, functions of radial transport and collection of photogenerated carriers need to be considered as well. Fig. 5 depicts the internal quantum efficiency (IQE) of RJ-HET and P-HET solar cells. It can be seen that IQE of RJ-HET solar cell is higher than that of P-HET solar cell within the short wavelength range (400-600 nm). It's known that short-wavelength incident light is mainly absorbed by SiNWs in RJ-HET solar cell. When wavelength $<450 \mathrm{~nm}$, absorption length is around $1 \mu \mathrm{m}$ in c-Si. Since average diameter of SiNWs (dozens of nanometer) is much less than the diffusion length of minority carries, photogenerated carriers can be collected rapidly in radial junctions of SiNWs solar cell, thus the short wavelength range of IQE increases in the RJ-HET solar cell. In the range of medium and long wavelength, IQE of RJ-HET solar cell is a little bit lower than that of P-HET solar cell, which may be related to differences of bulk and rear surface recombination.

Nanostructures acting as light trapping layer exhibit a superior antireflection characteristic, i.e. omnidirectionality. ${ }^{26-28}$ It's of great benefit to photovoltaic application if solar cell has the omnidirectionality. To exam omnidirectional antireflection properties of RJ-HET and P-HET solar cells, angulardependences of $V_{\mathrm{oc}}, J_{\mathrm{sc}}, \mathrm{FF}$, and apparent EFF were measured and compared as shown in Fig. 6. The experimental measured apparent efficiency describes the electrical output of the solar cell under different incident angle of the light. For P-HET solar cell, shown in Fig. 6(b), the light $I-V$ data strongly depend on the angle of incident (AOI). $J_{\mathrm{sc}}, V_{\mathrm{oc}}$ and apparent EFF decrease rapidly with AOI increasing. Compared to normal incidence condition, $J_{\mathrm{sc}}$ reduces by $11.9 \%$ and $21.9 \%$ as AOI changes to be

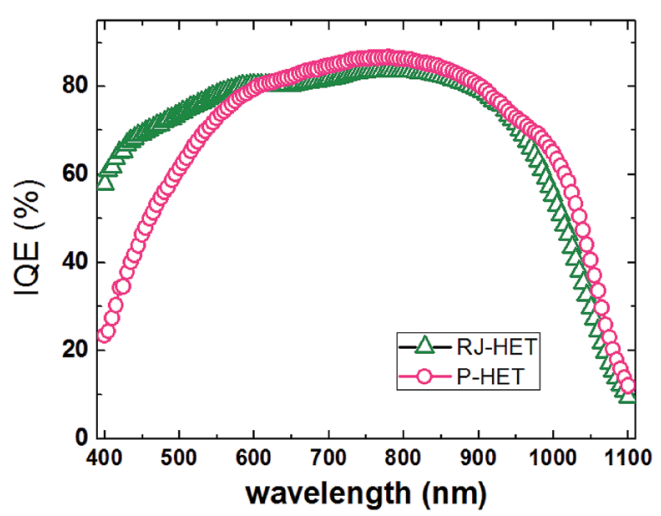

Fig. 5 IQE of RJ-HET and P-HET solar cells. The RJ-HET solar cell exhibits an enhanced short wavelength range spectral response, which confirms the validity and superiority of carrier collection in radial junctions.

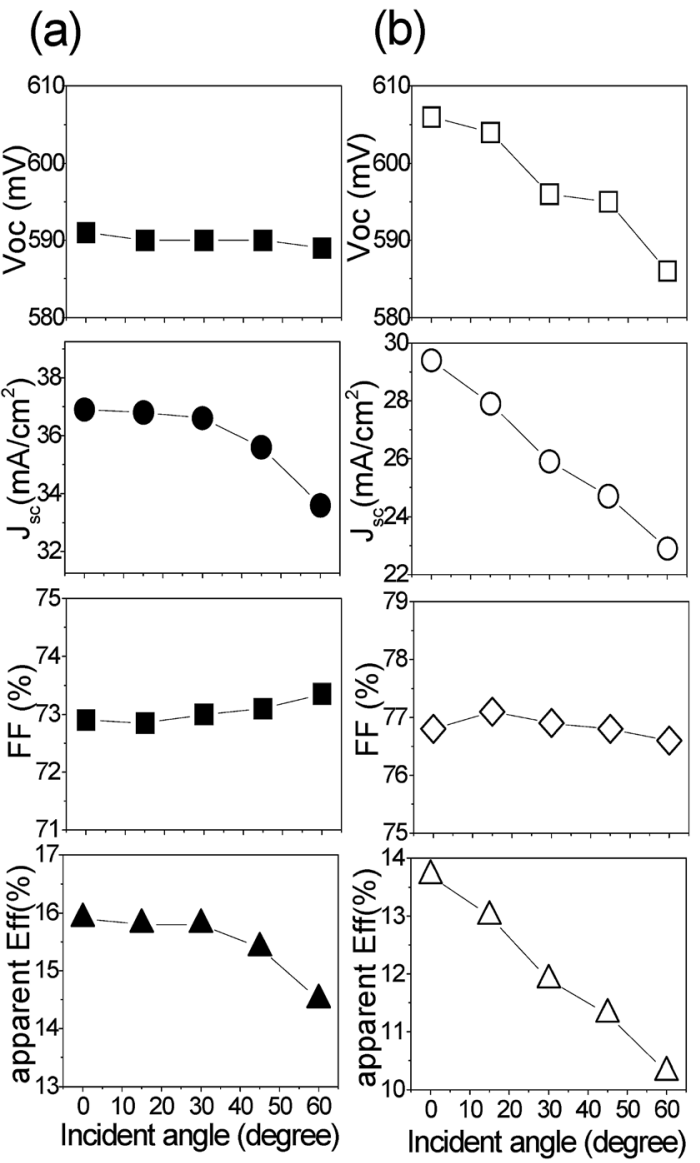

Fig. 6 Performances of (a) RJ-HET and (b) P-HET solar cells as a function of incident angle of light. The RJ-HET solar cell shows a much better omnidirectional antireflection property than the P-HET solar cell.

$30^{\circ}$ and $60^{\circ}$. Loss of $J_{\text {sc }}$ leads to low apparent EFF for the P-HET solar cell at large AOI conditions. In comparison, RJ-HET solar cell exhibits a better antireflection property over a wide AOI range. Light trapping effect of SiNWs arrays is correlated with incident light random scattering. Scattered and redirected light can be re-scattered in the SiNWs arrays. Variety of AOI has low impact on this multiple scattering process. As shown in Fig. 6(a), $V_{\mathrm{oc}}, J_{\mathrm{sc}}, \mathrm{FF}$ and apparent EFF are almost independent of AOI, although $J_{\mathrm{sc}}$ decreases slightly as AOI $>30^{\circ}$. The angulardependences of light $I-V$ dada indicate that RJ-HET solar cell with SiNWs structure is remarkably insensitive to AOI over a wide range. PV power station will benefit of this good omnidirectional light-harvesting ability of RJ-HET solar cell. In particular, the unique light-trapping characteristic and the advanced electric collection mode are suitable strategies to maintain competitively high efficiency of the ultrathin crystalline silicon solar cells while shrinking the volume of silicon.

\section{Conclusion}

In summary, a-Si : $\mathrm{H} / \mathrm{SiNWs} \mathrm{RJ}-\mathrm{HET}$ solar cells were fabricated by Cat-CVD combined with MACE. Nice conformal coating of a-Si : $\mathrm{H}$ thin film on MACE SiNWs with high aspect ratio and large density was realized. The optimal combination of average 
length of SiNWs and deposition time of a-Si : $\mathrm{H}$ was determined. Optimized thickness (deposition time) of intrinsic a$\mathrm{Si}$ : $\mathrm{H}$ layer is $10 \mathrm{~nm}$ and doped a-Si : $\mathrm{H}$ layer is $20 \mathrm{~nm}$ for SiNWs with $1 \mu \mathrm{m}$ length. The highest efficiency of $16.02 \%$ of RJ-HET solar cell was achieved. Capacitance-voltage and dark current-voltage measurements indicate that atomic $\mathrm{H}$ treatments and a-Si : $\mathrm{H}$ passivation by Cat-CVD reduce the surface recombination effectively and enable a higher $V_{\text {oc }}$ for RJ-HET solar cell compared with RJ-HOMO solar cell. $J_{\text {sc }}$ of RJ-HET solar cell increases 24-25\% compared with P-HET solar cell due to enhanced light absorption of SiNWs structure and radial collection of carriers. And, the radial collection of carriers has a significant contribution to shortwave response in IQE spectra. RJ-HET solar cell is remarkably insensitive to AOI over a wide range, which indicates an excellent omnidirectional antireflection property.

It should be pointed out that all fabrication processes were carried out in an open lab without air cleaning systems. The interface quality and efficiency of solar cells will certainly be improved if all processes went through in a clean room. In addition, the whole process temperature is below $200{ }^{\circ} \mathrm{C}$, which is suitable for fabrication of ultrathin c-Si solar cell. So, this novel RJ-HET solar cells based on MACE SiNWs and Cat-CVD techniques have great potential in application of highefficiency and low cost photovoltaic devices in the future.

\section{Conflicts of interest}

There are no conflicts to declare.

\section{Acknowledgements}

This work was supported by the National Natural Science Foundation of China (No. 61674150 and No. 61604153).

\section{References}

1 B. M. Kayes, H. A. Atwater and N. S. Lewis, J. Appl. Phys., 2005, 97, 114302.

2 Y. Kuang, M. D. Vece, J. K. Rath, L. V. Dijk and R. E. I. Schropp, Rep. Prog. Phys., 2013, 76, 106502.

3 B. Tian, X. Zheng, T. J. Kempa, Y. Fang, N. Yu, G. Yu, J. Huang and C. M. Lieber, Nature, 2007, 449, 885-889.

4 H. P. Yoon, Y. A. Yuwen, C. E. Kendrick, G. D. Barber, N. J. Podraza, J. M. Redwing, T. E. Mallouk, C. R. Wronski and T. S. Mayer, Appl. Phys. Lett., 2010, 96, 213503.

5 B. Dou, R. Jia, H. Li, C. Chen, W. Ding, Y. Meng, Z. Xing, X. Liu and T. Ye, Appl. Phys. Lett., 2012, 101, 183901.
6 G. Dong, F. Liu, J. Liu, H. Zhang and M. Zhu, Nanoscale Res. Lett., 2013, 8, 544.

7 J. Oh, H. C. Yuan and H. M. Branz, Nat. Nanotechnol., 2012, 7, 743-748.

8 X. Lin, X. Hua, Z. Huang and W. Shen, Nanotechnology, 2013, 24, 235402.

9 M. A. Green, K. Emery, Y. Hishikawa, W. Warta and E. D. Dunlop, Prog. Photovoltaics, 2017, 25, 3-13.

10 K. Yoshikawa, H. Kawasaki, W. Yoshida, T. Irie, K. Konishi, K. Nakano, T. Uto, D. Adachi, M. Kanematsu and H. Uzu, Nat. Energy, 2017, 2, 17032.

11 G. Jia, M. Steglich, I. Sill and F. Falk, Sol. Energy Mater. Sol. Cells, 2012, 96, 226-230.

12 K. Li, X. Wang, P. Lu, J. Ding and N. Yuan, Nanoscale Res. Lett., 2013, 8, 396.

13 E. S. Ashour, M. Y. B. Sulaiman, M. H. Ruslan and K. Sopian, Nanoscale Res. Lett., 2013, 8, 466.

14 F. Wang, Y. Jiang, T. Li, Y. Zhao and X. Zhang, J. Mater. Chem. A, 2015, 3, 22902-22907.

15 H.-P. Wang, T.-Y. Lin, M.-L. Tsai, W.-C. Tu, M.-Y. Huang, C.-W. Liu, Yu-L. Chueh and Jr-H. He, ACS Nano, 2014, 8(3), 2959-2969.

16 R. E. I. Schropp, Thin Solid Films, 2015, 595, 272-283.

17 R. E. I. Schropp, Thin Solid Films, 2009, 517, 3415-3419.

18 S. Osono, Y. Uchiyama, M. Kitazoe, K. Saito, M. Hayama, A. Masuda, A. Izumi and H. Matsumura, Thin Solid Films, 2003, 430, 165-169.

19 Q. Wang, S. Ward, L. Gedvilas, B. Keyes, E. Sanchez and S. Wang, Appl. Phys. Lett., 2004, 84, 338-340.

20 H. Matsumura, M. Miyamoto, K. Koyama and K. Ohdaira, Sol. Energy Mater. Sol. Cells, 2011, 95, 797-799.

21 Q. Wang, M. R. Page, E. Iwaniczko, Y. Xu, L. Roybal, R. Bauer, B. To, H.-C. Yuan, A. Duda, F. Hasoon, Y. F. Yan, D. Levi, D. Meier, H. M. Branz and T. H. Wang, Appl. Phys. Lett., 2010, 96, 013507.

22 A. Matsuda, Thin Solid Films, 1999, 337, 1-6.

23 C. E. Richardson, Y. B. Park and H. A. Atwater, Phys. Rev. B: Condens. Matter Mater. Phys., 2006, 73, 245328.

24 B. Jagannathan, W. A. Anderson and J. Coleman, Sol. Energy Mater. Sol. Cells, 1997, 46, 289-310.

25 R. Hussein, D. Borchert, G. Grabosch and W. R. Fahrner, Sol. Energy Mater. Sol. Cells, 2001, 69, 123-129.

26 P. Spinelli, M. A. Verschuuren and A. Polman, Nat. Commun., 2012, 3, 692.

27 W. Q. Xie, J. I. Oh and W. Z. Shen, Nanotechnology, 2011, 22, 065704.

28 C. A. Lin, K. Y. Lai, W. C. Lien and J. H. He, Nanoscale, 2012, 4, 6520-6526. 